\title{
Erratum to: Targeting $A L K$ : a promising strategy for the treatment of non-small cell lung cancer, non-Hodgkin's lymphoma, and neuroblastoma
}

Andres Morales La Madrid • Nicholas Campbell • Sonali Smith • Susan L. Cohn • Ravi Salgia

Published online: 8 September 2013

(C) Springer-Verlag France 2013

Erratum to: Targ Oncol (2012) 7:199-210

DOI 10.1007/s11523-012-0227-8

The original publication of this article contains a mistake.

The Family name of the first Author should be Morales La Madrid. 